Claremont Colleges

Scholarship@ Claremont

All HMC Faculty Publications and Research

HMC Faculty Scholarship

$1-1-1982$

\title{
Some Properties of Positive Derivations on f-Rings
}

Melvin Henriksen

Harvey Mudd College

Frank A. Smith

Kent State University

\section{Recommended Citation}

Henriksen, Melvin, and F. A. Smith. "Some properties of positive derivations on f-rings." Proceedings of the Special Session on Ordered Field and Real Algebraic Geometry, 87th Annual Meeting of the American Mathematical Society (San Francisco, CA, 7-11 January 1981). Ed. D. W. Dubois and T. Recio. Contemporary Mathematics 8 (1982): 175-184.

This Article is brought to you for free and open access by the HMC Faculty Scholarship at Scholarship @ Claremont. It has been accepted for inclusion in All HMC Faculty Publications and Research by an authorized administrator of Scholarship @ Claremont. For more information, please contact scholarship@cuc.claremont.edu. 


\section{INTRODUCTION}

Throughout A denotes an f-xing; that is, a lattice-ordered ring that is a subdirect union of totaliy ordered rings. We let $D(A)$ denote the set of derivations $D: A \rightarrow A$ such that $a \geq 0$ implies $D a \geq 0$, and we call such derivations positive. In [CDK], P. Coleville, G. Davis, and $K$. Keimel initiated a study of positive derivations on f-rings. Their main results are (i) $D \in D(A)$ and $A$ archimedean imply $D=0$, and (ii) if $A$ has an identity element 1 and $a$ is the supremum of a set of integral multiples of 1 , then $D a=0$. Their proof of (i) relies heavily on the theory of positive orthomorphisms on archimedean f-rings and gives no insight into the general case. Below, in Theorem 4 and its corollary, we give a direct proof of (i), and in Theorem 10, we generalize ( $i j$ ). Throughout, we improve on results in [CDK], and we study a variety of topics not considered therein.

\section{THE RESULTS}

In the sequel, $A$ will always denote an f-ring, and $A^{+}=\{a \in A: a \geq 0\} \quad i t s$ positive cone. If $a \in A$, let $a^{+}=a \vee 0$, $a^{-}=(-a) \vee 0$, and $|a|=a \vee(-a)$. Then $a=a^{+}-a^{-}, \quad|a|=a^{+}+a^{-}$, and $a^{+} a^{-}=a^{-} a^{+}=a^{+} \wedge a^{-}=0$. A subset $I$ of $A$ that is a ring ideal and such that $|b| \leq|a|$, and $a \in I$ imply $b \in I$ is called an $\ell$-ideat. The $\ell$-ideals are the kernels of homomorphisms that preserve lattice as well as ring operations [BKW, Chap. 8].

$A$ derivation on $A$ is a linear map $D: A \rightarrow A$ such that if $a, b \in A$, then $D(a b)=a D b+(D a) b$. A derivation $D$ is called positive if $D\left(A^{+}\right) \subset A^{+}$. The family of alt positive derivations on $A$ will be denoted by $D(A)$.

1980 Mathematics Subject Classification. 13N05, 06F25, 12J15, $13 \mathrm{~J} 25$. 
In any f-ring $\operatorname{rad} A$, the set of all nilpotent elements of $A$, coincides with the intersection of all the prime $l$-ideals of $A$, and hence is an $\ell$-ideal $[B K W, 9.2 .6]$. If $\operatorname{rad} A=\{0\}$, then $A$ is said to be reduced. In [COK], it is shown that if $A$ is commutative and $a^{n}=0$, then $[\mathrm{Da}]^{2 n-1}=0$. We improve this result next. We begin by observing that if $a, b, \in A^{+}$then

$$
a b=0 \text { implies } \quad a D b=(D a) b=0 .
$$

1. PROPOSITION. Suppose $a \in A$ and $D \in D(A)$. Then $a^{n}=0$ implies $(D a)^{n}=0 . \quad$ In particular, $D[\operatorname{rad} A] \subset \operatorname{rad} A$.

PROOF. Since $a^{n}=0$ if and only if $|a|^{n}=0$, we may assume $a \in A^{+}$and $n>1$. By (1), $a^{n-1} D a=0$. So $a^{n-2}(a D a)=0$. Using (1) again yields $0=a^{n-2} D(a D a)=a^{n-1} D^{2} a+a^{n-2}(D a)^{2}$. Sirce $a \in A^{+}$, $a^{n-2}(D a)^{2}=0$. Continuing this process yields $(D a)^{n}=0$ arid hence that $D[\operatorname{rad} A] \in \operatorname{rad} A$.

The next example will show that the index of nilpotency of Da need not be less than that of a. We note first that if $D \in D(A)$ and $I$ is an $\ell$-ideal of $A$ such that $D(I) \subset I$, then $D_{I} \in D(A / I)$, where

$$
D_{I}(a+I)=D a+I \text {, }
$$

2. EXAMPLE. Let $R$ denote all rational functions with real coefficients of negative degree. If $r(x)=\frac{p(x)}{q(x)} \in R$, we may assume that $q(x)=x^{m}+a x^{m-1}+\ldots$ has leading coefficient 1 , and we let $r(x)$ be positive if the leading coefficient of $p(x)$ is positive. With this order, $R$ is a totally ordered ring. If $r(x) \in R$, let $\operatorname{Dr}(x)=-r^{\prime}(x)$ be the negative of the usual derivative. Then $D \in D(R)$, as is $(x D): R \longrightarrow R$, where $(x D) r(x)=x \operatorname{Dr}(x)=-x r^{\prime}(x)$. If $n$ is a positive integer, let $I_{n}$ denote the set of all $r(x)$ in $R$ of degree $\leq-n$. Clearly $I_{n}$ is an l-ideal of $R$, and $(x D)\left(I_{n}\right)=I_{n}$. If $R_{n}=R / I_{n}$, and $(x D)_{n}\left(r(x)+I_{n}\right)=x \operatorname{Dr}(x)+I_{n}$, then $(x D)_{n} \in D\left(R_{n}\right)$. and $(x D)_{n}\left(\frac{I}{x}+I_{n}\right)=\frac{1}{x}+I_{n} \quad$ is nilpotent of index $n$.

If $G$ is an abelian $\ell$-group, and $T: G \rightarrow$ is an order preserving endomorphism of $G$ such that $x \wedge y=0$ implies $x \wedge$ Ty $=0$ for $x, y$ in $G^{+}$, then $T$ is called a positive orthomorphism of $G$. If $A$ is reduced, then $x \wedge y=0$ if and only if $x y=0$ [BKW, 9.3.1]. 
So each positive derivation on an f-ring is an orthomorphism by (1). The next result appears implicitly in [CDK]. We include a proof for the sake of completeness.

3. PROPOSITION. If $\mathrm{P}$ is a minimal prime $\ell$-ideal of $\mathrm{A}$, and $D \in D(A)$, then $D(P) \subset P$. In particular, $D_{P} \in D(A / P)$.

PROOF. As is noted in [BKW, 9.3.2 and 12.1.1], if $A$ is reduced, then each positive orthomorphism of $A(t)$ maps a minimal prime subgroup into itself, and $P$ is a minimal prime $l$-ideal of $A$ if and only if it is a minimal prime subgroup. So $D(P) \subset P$ if $A$ is reduced. In the general case, if we let $I=\operatorname{rad} A$ in $(2)$, we obtain $D(P) \subset P$.

We do not know if $D(P) \subset P$ for any prime $\ell$-ideal of $P$.

Recall that $A$ is said to be archimedean if $a \in A^{+}$and $\{n a: n=1,2, \ldots\}$ bounded above imply $a=0$. The next theorem is the key to an alternate proof of the fact that a reduced archimedean f-ring admits no nontrivia] derivations [CDK].

4. THEOREM. Suppose $A$ is reduced, $D \in D(A), a \in A^{+}$, and $n$ is a positive integer. Then
(a) $n\left(a \wedge a^{2}\right) D a \leq\left(a \vee a^{2}\right) D a$,
(b) $n D a\left(a \wedge a^{2}\right) \leq D a\left(a \vee a^{2}\right)$, and
(c) $\mathrm{nD}\left(\mathrm{a}^{2}\right) \leq\left(\mathrm{a}^{2} \mathrm{Da}+(\mathrm{Da}) \mathrm{a}^{2}\right) \vee \mathrm{Da}$.

PROOF. Since $A$ is reduced, $\{0\}$ is an intersection of minimal prime ideals and $A$ is a subdirect sum of totally ordered rings $A / P$ such that $P$ is a minimal prime $\ell$-ideal. Thus, by Proposition 3 , it suffices to verify these identities in case $A$ is totally ordered and has no proper divisors of 0 [BKW, 9.2.5].

Let $x=\left(n a-a^{2}\right)^{+} D a$. Then $x \in A^{+}$. We consider two cases:

(i) Suppose $x=0$. Then $D a=0$ or $n a \leq a^{2}$. In either case we obtain

$$
\text { naDa } \leq a^{2} D a \text { and } n(D a) a \leq(D a) a^{2} \text {. }
$$

(ii) Suppose $x>0$. Then $D a>0$ ard $a^{2}<$ na. Hence $a D a+(D a) a \leq n D a$. Since $A$ is totally ordered, $a D a \leq(D a) a$ or $(D a) a \leq a(D a)$.

Suppose the former holds. Then

$$
2 \mathrm{aDa} \leq \mathrm{nDa} \quad \text { and hence } \quad\left(n a-2 \mathrm{a}^{2}\right) \mathrm{Da} \geq 0 .
$$


But Da $>0$, so $2 a^{2} \leq n a$. By induction, we get $2^{k} a^{2} \leq n a$ for $k=0,1,2, \ldots$ If we choose $k$ so large that $n^{2} \leq 2^{k}$, we get

$$
n a^{2} \leq a \text {. }
$$

If, instead, (Da)a $\leq \mathrm{aDa}$, an obvious modification of this latter argument also yields (4). Pre or post multiplying by $\mathrm{Da}$ yields

$$
n a^{2} \mathrm{Da} \leq \mathrm{aDa} \text { and } \mathrm{n}(\mathrm{Da}) \mathrm{a}^{2} \leq(\mathrm{Da}) \mathrm{a} \text {. }
$$

Since either (3) or (5) must hold in $A / P$ for any minimal prime ideal $P$, the conclusions of (a) and (b) hold.
By (4), if $x>0$, then $n D\left(a^{2}\right) \leq D(a)$. If $x=0$, then adding the inequalities in (3) yields $\mathrm{nD}\left(\mathrm{a}^{2}\right) \leq\left(\mathrm{a}^{2} \mathrm{Da}+(\mathrm{Da}) \mathrm{a}^{2}\right)$. Hence (c) holds as well.

5. COROLLARY. [CDK] If $A$ is archimedean and $D \in D(A)$, then $D(A) \subset \operatorname{rad} A$ and $D\left(A^{2}\right)=0$.

PRO0F. By (c) of the last theorem and Proposition 3, if $a \in A$, then $D\left(a^{2}\right) \in \operatorname{rad} A$. Since $a D a \leq D\left(a^{2}\right), \quad(D a)^{2} \leq D(a D a) \leq D^{2}\left(a^{2}\right) \in D(\operatorname{rad} A)$ $C \operatorname{rad} A$ by Proposition 1. Since each element of $\operatorname{rad} A$ is nilpotent, so is Da.

If $a, b \in A$, then $D(a b)=a D b+(D a) b=0$, since $(\operatorname{rad} A) A=A(\operatorname{rad} A)=0$ in an archimedean f-ring $[B K W, 12.3 .11]$. Hence $D\left(A^{2}\right)=0$.

6. PROPOSITION. Suppose $e^{2}=e \in A$ and $D \in D(A)$.

(a) $(D e)^{2}=e(D e) e=(D e) e(D e)=0$.

(b) If $A$ is reduced or has an identity element or $e$ is in the center of $A$, then $D e=0$.

PROOF. Since $e^{2}=e$, we have

$$
\mathrm{eDe}+(\mathrm{De}) \mathrm{e}=\mathrm{De}
$$

Muttiplying (6) on the left by e yields

$$
e(D e) e=0 \text {. }
$$

Applying D to (7), we obtain

$$
e D[(D e) e]+(D e)^{2} e=0=e(D e)^{2}+D(e D e) e .
$$


Hence

$$
e(D e)^{2}=(D e)^{2} e=0
$$

Multiplying both sides of (6) on the left by (De) and using (8)

yields

$$
(\mathrm{De}) \mathrm{e}(\mathrm{De})=(\mathrm{De})^{2} \text {. }
$$

By (7), (8), and (9), we obtain

$$
[e D e-(D e) e]^{2}+(D e) e(D e)=0 .
$$

Hence $(\mathrm{De})^{2}=(\mathrm{De}) \mathrm{e}(\mathrm{De})=0$, which together with $(7)$, completes the proof of $(a)$.

Clearly $D e=0$ if $\operatorname{rad} A=\{0\}$. If $\mathrm{eDe}=$ (De)e, then by (6) and (7), $\quad \mathrm{De}=2 \mathrm{eDe}=0$. If $\mathrm{A}$ has an identity element, then each of its idempotents is in the center of $A$ by [BKW, 9.4.20]. This completes the proof of (b).

The next example shows that the hypotheses of (b) above cannot be omitted.

7. EXAMPLE. A totalzy ordered ring with an idempotent $e$ and a positive derivation $D$ such that $\mathrm{De} \neq 0$.

Let $S$ denote the algebra over the real fieid $\mathbb{R}$ (with the usuat order) with basis $\{e, z\}$, where $e^{2}=e, e z=z^{2}=0$, and $z e=z$. If $x=\alpha e+\beta z \in S$, let $x>0$ if $\alpha>0$ or $\alpha=0$ and $\beta>0$. If we let $D x=z x-x z=\alpha z$, then $D \in D(S)$, and De $=z \neq 0$.

If $D \in D(A)$, let $\operatorname{ker} D=\{a \in A: D a=0\}$. If $G$ is an abelian $\ell$-group and $H \subset G$, let $H^{\perp}=\{g \in G:|g| \wedge|h|=0$ for $a 11$ $h \in H\}$, and let $H^{\perp \perp}=\left(H^{\perp}\right)^{\perp}$. Note that $H^{\perp}$ is an $\ell$-subgroup of $G$ (that is, $H$ is a subgroup and $|a| \leq|b|$, and $b \in H^{\perp}$ implies $a \in H$ ). A band in $G$ is an $\ell$-subgroup $H$ of $G$ such that if $K \in H$ and $\sup K \in G$, then $\sup K \in H$. If $H$ is a subset of $G$, the intersection $B(H)$ of all the bands in $G$ containing $H$ is also a band. Moreover, $B(H) \subset H^{1 \perp}$. See [LZ, Theorem 19.2I. An element $e$ of $G$ such that $\{e\}^{\perp}=0$ is called a weak order unit of $G$. An element $e$ of an f-ring $A$ such that ex $=0$ or $x e=0$ implies $x=0$ is called reguzar. Note that if $e \in A$ is regular, then $e$ is a weak order unit, and the converse holds if $A$ is reduced. 
The following lemma will be useful in what follows.

8. LEMMA. Suppose $A$ is an $f$-ring and $D \in D(A)$.

(a) $\mathrm{xDx} \wedge(\mathrm{Dx}) \mathrm{x} \geq 0$ for every $\mathrm{x} \in \mathrm{A}$.

(b) If $A$ is reduced, then $D$ is an l-endomorphism.

(c) If $\mathrm{A}$ has an identity element 1 , and $\mathrm{n}$ is a positive integex, then $\mathrm{nDx} \leq \mathrm{XDx} \wedge(\mathrm{Dx}) \mathrm{x}$ for every $\mathrm{x} \in \mathrm{A}^{+}$and $D(I) \subset I$ for every l-ideal I of $A$.

PROOF. (a) holds since this inequality holds whenever $A$ is totally ordered.

(b) holds since if $A$ is reduced, then $D$ is a positive orthomorphism and hence an $\ell$-endomorphism [BKW, 12.1].

(c) by Proposition 6(b), $1 \in \operatorname{ker} D$, and by $(a)(x-n T) D(x-n 1) \geq 0$. Hence $n D x \leq x D x$. Similarty, $n D x \leq(D x) x$. Hence $x \in I$ implies $D x \in I$ since $I$ is an $\ell$-ideal.

Next, we provide some examples to show that the hypotheses of (b) and (c) above cannot be omitted.

9. EXAMPLES. (i) Let $E$ denote the direct sum of two copies of the real line $\mathbb{R}$ with trivial multiplication, and let $(r, s) \geq 0$ mean $r \geq s \geq 0$. As is noted in $[G J, 5 B]$, the map $D: E \longrightarrow E$ such that $D(r, s)=(r, 0)$ is a positive endomorphism that is not an $\ell$-homomorphism. To see the latter, note that $(1,2)^{+}=(2,2)$. So $\mathrm{D}\left[(1,2)^{+}\right]$ $=(2,0) \neq(1,0)=\left[D(1,2]^{+}\right.$.

(ii) Let $R$ and $(x D)$ be as in Example 2, and let $y=\frac{1}{x}$. Then $n(X D) y=\frac{n}{x}$, while $y(x D) y=x^{-2}$, so the conclusion of (c) fails.

The next theorem summarizes most of what we know about kernels of positive derivations.

10. THEOREM. Suppose $D \in \mathcal{D}(\mathrm{A}), \mathrm{x} \in \mathrm{A}$, and $\mathrm{n}$ is a positive integer.

(a) If $\mathrm{e}$ is reguzar, and ex $\in$ ker $\mathrm{D}$, then $\mathrm{x} \in \mathrm{ker} \mathrm{D}$.

(b) If $\mathrm{A}$ is reduced then:

(i) $x \in \operatorname{ker} D$ implies $\{x\}^{1 \perp} \in \operatorname{ker} D$,

(ij) $x^{n} \in \operatorname{ker} D$ implies $x \in \operatorname{ker} D$,

(iii) $\operatorname{ker} D$ is a band, 
(iv) $D^{n}=0$ implies $D=0$, and

(v) $e^{2}=e \in A$ implies $e \in \operatorname{ker} D$.

(c) If $A$ has an identity element and $U(A)$ is the smaliest band containing the units of $A$, then $U(A) \subset$ ker D. In particular, $\operatorname{rad} A \subset \operatorname{ker} D$. Also, if $x^{2} \leq x$, then $x \in \operatorname{ker} D$.

PROOF. (a) By (1), $D(e x)=0$ implies $e D x=0$, which, in turn implies $\mathrm{Dx}=0$.

(b) (i) By Lemma $8(b)$, and $[B K W, 3.2 .2], D\left(\{x\}^{\perp \perp}\right) \subset D\left(\{x\}^{\perp}\right)^{\perp}$ $c\{(D x)\}^{11}=\{0\}$ since $x \in \operatorname{ker} D$ and $A$ is reduced.

(ii) follows from (i) and the fact that $\{x\}^{\perp \perp}$ is the intersection of all the minimal prime $\ell$-ideals that contains $x$ [BKW, 3.4.12].

(iii) As was noted above, the smallest band containing ker $D$ is contained in $\{(\text { ker } D)\}^{\perp \perp}$ and the latter is contained in ker $D$ by $(i)$.

(iv) Since $x$ is a difference of positive elements, it suffices to show that $D x=0$ whenever $x \in A^{+}$. The proof will proceed by induction on $n$. It is obvious when $n=1$. Assume that $D^{n}(A)=0$ implies $D(A)=0$ whenever $A$ is a reduced f-ring and $n \geq 1$ is an integer. If $0=D^{n+1}(A)=D^{n}(D(A))$, then $D^{n}\left(D(A)^{ \pm \perp}\right)=0$ by (i). So $D\left(D(A)^{1 \perp}\right)=0$ by the induction hypothesis. In particular, $D^{2}\left(x^{2}\right)=0$. Since $\quad x D x \leq D\left(x^{2}\right), \quad 0=D(x D x)=x D^{2} x+(D x)^{2}$. So $(D x)^{2}=0=D x$ since $A$ is reduced.

(v) is a restatement of Proposition $16(b)$.

(c) That $u(A) \subset \operatorname{ker} D$ follows directly from (a) and (b) (iii) above. If $x^{n}=0$, then $(1-x)\left(1+x+\cdots+x^{n-1}\right)=1$, so $1-x$ is a unit and $x=1-(1-x) \in u(A) \subset$ ker $D$. Finally, if $x^{2} \leq x$, then $D\left(x^{2}\right)=x D x+(D x) x \leq D x \leq x D x \wedge(D x) x$ by Lemma $8(c)$. Hence $x D x=(D x) x=0$. Thus $D x=0$. This completes the proof of Theorem 10 .

11. EXAMPLES AND REMARKS. The assumption that $A$ is reduced in Theorem 10(b) cannot be dropped. For example, if $A=C[0,1]$, the $\ell$-group of continuous real-valued functions on $[0,1]$, with trivial multiplication for all $f \in C[0,1]$, we let $D f=f\left(\frac{l}{2}\right)$, then $D \in D(A)$, and $\operatorname{ker} D$ fails to be a band [DV, p. 12]. Also, the plane $E^{2}$ with the usual coordinatewise addition and trivial multiplication admits positive endomorphisms that are nilpotent. (For example, let $T(a, b)=(0, a)$ for $\left.a\rceil 1,(a, b) \in E^{2}\right)$. Theorem 10(c) genera]jzes [CDK, Theorem 7] where it is shown that ker D contains the supremum of any set of elements bounded above by some integral multiple of the identity element. 
As in $[P]$, we let $I_{0}(A)=\left\{a \in A: n|a| \leq x\right.$ for some $x \in A^{+}$ and $n=1,2, \ldots\}$. Clearly $I_{0}(A)$ is an $\ell$-ideal and $I_{0}(A)=\{0\}$ if and only if $A$ is archimedian.

12. THEOREM. Suppose $D \in D(A)$.

(a) If $A$ is reduced, then $D\left(A^{2}\right) \subset I_{0}(A)$.

(b) If $A$ has an identity element, then $\mathrm{D}(\mathrm{A})=\mathrm{I}_{0}(\mathrm{~A})$. If, moreover, $A$ is reduced and $I_{0}(A) \subset u(A)$, then $D=0$.

PRO0F. (a) follows immediately from Theorem 4 and the fact that $a b \leq(a \vee b)^{2}$ whenever $a, b \in A^{+}$.

(b) That $D(A) \subset I_{0}(A)$ is a restatement of Lemma $10(\mathrm{c})$. If $I_{0}(A) \subset U(A)$, then by Theorem $10(c), \quad D^{2}(A) \subset D(U(A))=\{0\}$. Hence if $A$ is reduced, then $D=0$ by Theorem $10(b)$.

13. EXAMPLES AND REMARKS.

(a) The reader may easily verify for the f-ring $R$ of Example 2, $I_{0}(R)=I_{2}$, while $(x D)(R)=R$. So the hypothesis in Theorem $12(b)$ that $A$ has an identity element may not be dropped if we wish to have $D(A) \subset I_{0}(A)$.

(b) Let $S$ denote the ring of all functions of the form

$$
\sum_{i=0}^{n} a_{i} x^{r}
$$

where $a_{i}$ is an integer and $r_{i}$ is a nonnegative rational number, ordered lexicographically, with the coefficient of the largest power of $x$ dominating. Then $I_{0}(S)=S$, and $U(A)$ is the set of constant polynomials. So, the condition of Theorem 12(b) fails. Despite this,

$D \in D(S)$ implies $D=0$.

For if $D \in D(S)$, then $D(x)=D\left(\left(x^{1 / 2}\right)^{2}=2 x^{1 / 2} D\left(\left(x^{1 / 4}\right)^{2}\right)\right.$ $=4 x^{3 / 4} D\left(\left(x^{1 / 8}\right)^{2}\right)=\cdots=2^{n} x^{7-1 / 2 n} D\left(x^{1 / 2 n}\right)$. Hence $2^{n} \mid D(x)$ for $n=0,1,2, \ldots$. Since the coefficients of any element of $S$ are integers, it follows that $D(x)=0$. A similar argument will show that $x^{r} \in$ ker $D$ whenever $r$ is a nonnegative rational number. It follows that $D=0$. We do not, however, know of any such example that is an algebraa over an ordered field. If $S^{*}$ is the result of allowing the coefficients of the elements of $S$ to be arbitrary rational numbers, and we let $D\left(x^{r}\right)=r x^{r}$ for any positive rational number $r$, then $D$ is a positive derivation. To see why, map $x^{r}$ to $e^{r x}$ and note that $S^{*}$ is isomorphic as an ordered ring to a subring of the ring of exponential polynomials, and the usual derivative on the latter maps the image of $S^{*}$ into itself. 
Our last result applies more general theorems and techniques of Herstein $\left[\mathrm{H}_{1}\right]\left[\mathrm{H}_{2}\right]$ to the context of positive derivations.

14. THEOREM. Suppose $A$ is reduced and $D \in D(A)$.

(a) If $D \neq 0$, then the ring $S$ generated by $\{\mathrm{Da}: \mathrm{a} \in \mathrm{A}\}$ contains a nonzero ideal of $A$.

(b) If $\mathrm{S}$ is commitative, then $\mathrm{S}$ is contained in the center of $A$.

(c) If $z \in A$ commutes with every element of $S$, $(a z-z a) \in \operatorname{ker} D$ for every a $\in A$. If, in addition, A is totally ordered and $D \neq 0$, then $z$ is in the center of $A$.

PROOF. (a) It is shown in $\left[\mathrm{H}_{\mathrm{j}}\right]$ that the conclusion holds for any derivation on any ring if $D^{3} \neq 0$. Since $A$ is reduced, $D^{3} \neq 0$ implies $D \neq 0$ by Theorem $10(b)$.

(b) Suppose $a \in S$ and $x \in A$. Then

$0=(D a) D(a x)-D(a x)(D a)=D a[a D x+(D a) x]-[a D x+(D a) x] D a=D a[(D a) x-x(D a)]$.

By $\left[\mathrm{H}_{3}\right.$, Lemma 1.1.4], Da is in the center of $\mathrm{A}$.

(c) The second statement is shown in $\left[\mathrm{H}_{2}\right]$, and the first follows immediately from the second and Theorem 10 (b).

\section{BIBLIOGRAPHY}

[BKW] A. Bigard, K. Keimel and S. Wolfenstein, Groupes et Anneaux Reticuzes, Lecture Notes in Mathematics 608, Springer-Verlag, New York, 1977.

[CDK] P. Colvil]e, G. Davis and K. Keimel, Positive derivations on f-rings, J. Austral Math. Soc. 23 (1977), 371-375.

[DV] E. De Jonge and A. Van Rooij, Introduction to Riesz Spaces, Mathematical Center Tracts 78, Amsterdam, 1977.

[GJ] L. Gillman and M. Jerison, Rings of Continuous Functions,

D. Van Nostrand Co., Pririceton, New Jersey, 1960.

[H] I. Herstein, A note on derivations, Canad. Math. Bul1. 21 (1978), $369-370$.

[H2] A note on dexivations II, ibid., 22 (1979), $509-511$

[H3] Rings with Involution, University of Chicago Press, Chicago, ITTinois, 1976.

[LZ] W. Luxemburg and A. Zaanen, Riesz Spaces I, North Holland Pub]. Co., Amsterdam, 1971. 
[P] J. Pairo, Yosida-Fukamiya's theorem for f-rings, pre-print.

HARVEY MUDD COLLEGE

CLAREMONT, CALIFORNIA 91711

KENT STATE UNIVERSITY

KENT, OHIO 44242

PITZER COLLEGE

CLAREMONT, CALIFORNIA 91711 